\title{
Aroma characterization of grape juice enriched with grapevine by-products using thermomaceration
}

\author{
Rafael Martinez ${ }^{1}$, Natalia Valderrama ${ }^{2}$, Juan Moreno ${ }^{1}$, and Johannes de Bruijn ${ }^{2 *}$
}

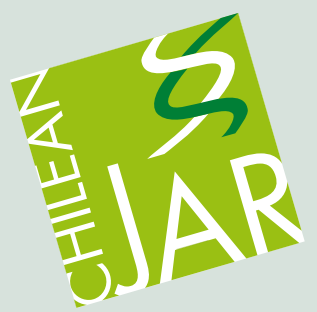

\section{ABSTRACT}

The need to reuse grapevine (Vitis vinifera L.) byproducts avoiding negative environmental impact demands the search for new valorization methods like thermomaceration. The combination of grapevine byproducts and grape must may be an alternative to get hold of additional aroma. The objective of this study was to assess the aroma potential of grape pomace, grapevine leaves and canes for País (PA) and Lachryma Christi (LC) cultivars to enrich grape must. Fifty aroma compounds were identified in the samples using stir bar sorptive extraction followed by thermal desorption, gas chromatography and mass spectrometry. 'País' must showed high contents of esters with a characteristic fruity odor, while benzenoids prevail in LC must giving a phenolic, balsamic odor. Thermomaceration increased contents of 13 free and bound volatile compounds, as well as new compounds (isoeugenol, phenol, vanillin and 2-ethyl hexanol) appeared in enriched juice (EJ). Cluster analysis showed differences among PA, LC and EJ samples. Principal component analysis was successfully applied to discriminate grape juice samples $(\mathrm{p}<0.05)$ being D-limonene, 5-hydroxymethyl-2-furfural and hexadecanoic acid the compounds that contributed most to the differentiation and increase of free and hydrolyzed aroma compounds after thermomaceration. Both grapegrapevine by-products and juice processing conditions affected the generation and increase of free and bound aroma compounds after thermomaceration of grape must.

Key words: Aroma compounds, aroma enrichment, chemometrics, grape must, thermomaceration, Vitis vinifera.

${ }^{1}$ Universidad de Córdoba, Facultad de Ciencias, Departamento de Química Agrícola y Edafología, Campus de Excelencia Internacional en Agroalimentación CeiA3, Ctra. N-IV-A, km 396, Córdoba, España. ${ }^{2}$ Universidad de Concepción, Facultad de Ingeniería Agrícola, Av. Vicente Méndez 595, Chillán, Chile.

"Corresponding author (jdebruij@udec.cl).

Received: 3 February 2017.

Accepted: 6 June 2017.

doi:10.4067/S0718-58392017000300234

\section{INTRODUCTION}

Despite the existence of many Vitis vinifera L. cultivars in wine producing countries, only a few cultivars account for the vast majority of worldwide wine production. Ancient grapevine cultivars played a significant role in many viticulture regions, but nowadays these cultivars cannot fulfil current quality standards of fine wines. Therefore, it is crucial to look for new opportunities for these wine grapes by the development of new grape berry based products. Considering the increased consciousness of many consumers about the importance of functional foods in their diet, the production of natural grape juice from undervalued, ancient grapevine cultivars may be an excellent opportunity for the viticulture sector. The consumption of pure fruit juices appreciated due to their healthy, natural and tasty characteristics is an easy and convenient way to secure one of the five portions of fruits and vegetables following the recommendations of the World Health Organization. Concerning the positive health benefits of the consumption of grape juice, an annual growing demand for grape juice of $2.75 \%$ is expected with an estimated average volume of $2.22 \mathrm{~L}$ per capita in 2020 (Statistica, 2016).

In addition to grape juice production, the generation of agroindustrial by-products, such as skins, seeds and stems, potential sources of health promoting phytochemicals, which can make up to $30 \%(\mathrm{w} / \mathrm{w})$ of raw material, has led to a growing concern in manufacturers (Teixeira et al., 2014). On the other hand, the valorization potential of these by-products as a source of aroma compounds for grape juice has been almost unexploited until now, which offers new opportunities. Grape skins have been found to contain more than half the free and glycoconjugated volatile compounds present in grape berries, being particularly rich in monoterpenes, norisoprenoids and aldehydes (Noguerol-Pato et al., 2012; Pedroza et al., 2013; Slegers et al., 2015). Grapevine matter other than grapes is a rich source of volatile compounds. Petioles contain terpenes with increased floral aroma (Ward et al., 2015); grapevine leaves are a source of carotenoids regarded as precursors of norisoprenoids (Baumes, 2009), while woody parts supplying volatile phenols (Weldegergis et al., 2011).

Cold maceration using dehydrated grape skin residues from the juice industry has been proposed by Pedroza et al. (2013) as a new enological tool to compensate color and aroma degradation in red wines. Moreover, skin maceration on its own juice in presence of sulfur dioxide using glycolytic enzymes to improve the availability of bound floral aroma compounds from berry 
skins may protect and improve flavor complexity (Pedroza et al., 2010; Noguerol-Pato et al., 2012). Additionally, heating and $\mathrm{pH}$ adjustment are appropriate techniques in juice processing to induce flavor in grape must by the hydrolysis of bound aroma precursors. However, hot break processing should be used carefully, especially with immature fruit, as it may result in the persistence of green aromas in grape juice (Iyer et al., 2010). Recently, thermomaceration has been used as a green extraction technique to fortify grape juice with antioxidant compounds from grape pomace, grapevine leaves and canes (Aguilar et al., 2016). Nevertheless, the potential of winery and grapevine by-products as a source of aroma compounds has still received small attention.

Therefore, the aim of this study was to assess the aroma potential of grape pomace, grapevine leaves and canes from País and Lachryma Christi cultivars to enrich grape musts using chemometrics. Additional knowledge about the volatile composition of grape musts with and without thermomaceration regarded as free and bound glycoside terpenoids, norisoprenoids, benzenoids, aldehydes, ketones, alcohols, acids and esters offers a means of evaluation of the possibilities of both grape cultivars to be used as raw material different to wine making.

\section{MATERIALS AND METHODS}

\section{Cultivars and grape-growing areas}

Grapes, leaves and canes from Vitis vinifera L. 'País' (PA), a red cultivar, and 'Lachryma Christi' (LC), a Teinturier cultivar, were provided by two vineyards located in the Itata Valley $\left(36^{\circ} 33^{\prime} \mathrm{S}, 72^{\circ} 10^{\prime} \mathrm{W}\right.$ and $36^{\circ} 30^{\prime} \mathrm{S}, 72^{\circ} 05^{\prime}$ W, respectively), San Nicolás, Chile, between April and June 2014. 'País' is an ancient Spanish cultivar also called 'Moscatel Negro', a Spanish homonym of 'Muscat' and synonym of 'Listán Prieto', now restricted to the Canary Islands in Europe. Moreover, it is known as 'País', Uva Negra Vino, Viña Blanca and Viña Negra in Chile, Criolla Chica in Argentina, Rosa del Peru and Negra Corriente in Peru, Misión in Mexico, Mission in United States, and Hariri in Morocco (Milla Tapia et al., 2007). 'Lachryma Christi' is a dyer or 'Teinturier', which accumulates red, purple pigments in pulp and skin, and commonly used for blending with pale red wine to give an intensive red color.

\section{Grape must and enriched juice preparation}

Healthy grapes were collected without reaching complete industrial maturity (sugar contents $169 \mathrm{~g} \mathrm{~L}^{-1}, \mathrm{pH} 3.03$ and total acidity $3.64 \mathrm{~g} \mathrm{~L}^{-1}$ ), then destemmed, crushed (PAS.0540, Bertuzzi, Brugherio, Italy) and pressed (D.64625, Willmes, Bensheim, Germany) to get must and pomace (skins and seeds). Afterwards pomace and must, previously treated by ultrasound-assisted cold pasteurization (Ultrasonic Cleaner 2800, Branson Ultrasonics, Danbury, Connecticut, USA) at $100 \mathrm{~W}$ for $30 \mathrm{~min}$, were stored at $-20{ }^{\circ} \mathrm{C}$ before processing and analysis. After vintage, autumn leaves and canes were cut and stored at $-20{ }^{\circ} \mathrm{C}$ until processing. Compounds from grape pomace, grapevine leaf and cane samples were extracted by thermomaceration as previously reported (Aguilar et al., 2016). After filtration using 20-25 $\mu \mathrm{m}$ nylon filter bags, extracts of pomace, leaves and canes were mixed in a proportion of 40:20:40\% (v/v/v), respectively. Enriched grape juice (EJ) compromised a blend of $250 \mathrm{~mL}$ extracts of both 'País' and 'Lachryma Christi' grapes, followed by heating $\left(63{ }^{\circ} \mathrm{C}\right.$ for $30 \mathrm{~min}$ ) and storage at room temperature until analysis.

\section{Extraction and chemical analysis of aroma compounds}

Free aroma fractions of grape musts and enriched juice were concentrated by stir bar sorptive extraction (SBSE) using a Twister coated with polydimethylsiloxane followed by thermal desorption (TD) and gas chromatography (GC) coupled to mass spectrometry (MS), using a previously validated method (Vararu et al., 2015). The analytical platform of SBSE-TD-GC-MS included: a thermal desorption unity (Gerstel GmbH, Mülheim an der Rühr, Germany) and an Agilent 7890A gas chromatograph equipped with a mass selective detector (5975, Agilent Technologies, Wilmington, Delaware, USA). The fused silica capillary column used was a HP-5MS $(30 \mathrm{~m} \times$ $0.25 \mathrm{~mm}$ i.d. $\times 0.25 \mu \mathrm{m}$ film thickness) from Agilent Technologies. Ethyl nonanoate was used as internal standard for determining the relative area from the total ion chromatogram peak area for each compound. Resulting data set is a semi-quantitative measure of the content of each compound. Further details about the extraction procedure, GC and mass detector operation conditions are given by Vararu et al. (2015).

Bound aroma compounds of grape musts and enriched juice were hydrolyzed by the addition of $2 \mathrm{M}$ citric acid to pH 2.5 according to Pedroza et al. (2010). After hydrolysis, the samples were cooled at room temperature using the same protocol as free aroma fraction analysis to determine the total content of aroma compounds (free and bound fractions).

Identification of the volatile compounds was done by comparing mass spectra with mass spectral data from the Wiley7N and NIST08 libraries, and confirmed by comparing the linear retention indices (LRI) calculated according to Vararu et al. (2015) with those standards compiled in the NIST Chemistry WebBook (NIST, 2016). Some compounds were also identified by comparing their mass spectrum with those from commercial standards available in our laboratory.

\section{Chemical standards and reagents}

A C7-C40 mixture in hexane (Sigma-Aldrich Quimica SL, Madrid, Spain) was used for LRI determination. Pure water was obtained from a Milli-Q purification system (Millipore, Madrid, Spain). All reagents and standards were of analytical grade and supplied by Fluka (Buchs, Switzerland), Merck (Darmstadt, Germany), Riedel de Haën (Seelze, Germany) or Sigma-Aldrich. 


\section{Statistical analyses}

The data of peak areas of three replicates for target compounds relative to the internal standard peak area, calculated after SBSE-TD-GC-MS analysis, were averaged and standard deviation (SD) was calculated. Data were previously normalized $\left(\log _{10} \mathrm{x}\right)$ and subjected to scaling techniques to avoid differences between units for each quantified compound (Seisonen et al., 2016). ANOVA and Fisher's least significant difference (LSD) method applied to the relative peak areas at 5\% significance level $(\mathrm{p}<0.05)$ were used in order to identify homogeneous groups (HG) and to determine the suitability of volatile compounds data for the differentiation between must and juice samples. Cluster analysis (CA) was carried out by calculating Euclidean distance for previously selected aroma compound data using Ward's minimum variance method to identify similarity among must and juice samples. Multiple variable analysis (MVA) was done by using the sum of the relative peak area for each individual compound for the major chemical groups as variable in order to characterize samples by finger printing their volatile compounds. Principal component analysis (PCA) was performed based on the relative area of selected aroma compounds as variables by selecting a number of linear combinations that account for most of the variability in data to visualize differences among the samples. The software package Statgraphics Plus, 2.0 (STSC Inc., Rockville, Maryland, USA) was used for statistical analysis.

\section{RESULTS AND DISCUSSION}

\section{Identification of volatile compounds}

Table 1 lists the mass spectrum match quality (MS Q) value, LRI calculated at experimental conditions, those compiled in the Wiley and NIST libraries and the Chemical Abstracts Service number of volatile compounds detected in must and juice samples of PA and LC grapes. Moreover, the providers of pure reagents are listed in this table. Establishing MS $Q$ values higher than 75 and LRI differences lower than 13 units as evaluation criteria, the identification of each compound can be considered as correct (Welke et al., 2014a). Fifty compounds were detected in the characteristic volatile profile of PA and LC samples, among them 34 species were unambiguously identified (Table 1). These compounds could be divided in nine chemical classes with their characteristic aroma descriptors: Terpenoids (two compounds), norisoprenoids (three compounds), lactones (two compounds), aldehydes and ketones (six compounds), alcohols (six compounds), benzenoids (nine compounds), alkenes (one compound), acids (eight compounds) and esters (13 compounds). The identified components have been reported before in grape and grape-derived products (Weldegergis et al., 2011; Ferrandino et al., 2012; Galano et al., 2015; Ghaste et al., 2015; González-Barreiro et al., 2015; Vararu et al., 2015; 2016; Yuan and Qian, 2016; Wang et al., 2017).

\section{Enrichment of aroma compounds}

Thermomaceration of grape pomace, grapevine leaves and canes in must was able to increase the content of free furanderived (2(5H)-furanone (6), 5-hydroxymethyl-2-furfural (13) and 2-furanmethanol (14)) and benzene-derived (phenol (21), 2-methoxy-4-vinylphenol (24), vanillin (25) and isoeugenol (26)) compounds (Table 2). Volatile benzenoids identified as isoeugenol, phenol and vanillin, were absent in the aroma fractions of PA and LC musts, so their origin in enriched grape juice can be attributed to thermomaceration of grape pomace, grapevine leaves and canes. On the other hand, total content of the following free and bound volatile compounds increased in enriched juice compared to grape musts: D-limonene (1), 1,1,6-trimethyl-1,2dihydronaphthalene (TDN) (4), 5-hydroxymethyl-2-furfural (13), 2-ethyl hexanol (16), benzaldehyde (20), isoeugenol (26), hexadecanoic acid (36) and 9,12-octadecadienoic acid (37) (Table 3). Except D-limonene, 5-hydroxymethyl-2furfural and isoeugenol, the other species are mainly present in glycosylated combinations. Amongst them, 2-ethyl hexanol is a new formed alcohol compound, detected after thermomaceration in enriched juice.

Thermal processing conditions may induce chemical reactions or inactivate enzymatic transformations affecting organoleptic properties of grape juice. Furan derivatives may be formed from sugars and furfural during heating in presence of woody material contributing to the burnt sugarlike aroma of enriched juice (Galano et al., 2015; Yuan and Qian, 2016). In addition, hot-press juice processing increased significantly the concentration of herbaceous odorant C6 alcohols (hexanol, cis-3-hexenol and trans-2hexenol) (Iyer et al., 2010). C6 alcohols that resulted from enzymatic degradation of unsaturated fatty acids or from glycoside aroma precursors were derived mainly from grapes, but also related to the presence of woody parts and leaves during grape crushing (Baumes, 2009; Yuan and Qian, 2016). Both grape skins and other matter than grapes can provide important fractions of total volatile compounds present that may affect sensory and chemical profiles of grape juice. In particular, skins contain relatively high concentrations of free monoterpenes, norisoprenoids, aldehydes and volatile phenols (Noguerol-Pato et al., 2012). Free volatile phenols like vanillin and isoeugenol may be directly recovered from grape skins (NoguerolPato et al., 2012; Slegers et al., 2015) or formed as byproducts of the lignin breakdown during thermomaceration of wood related material (Martínez-Gil et al., 2011; Weldegergis et al., 2011). Additionally, grapevine leaves may release terpenoids and benzenoids into grape juice (Ward et al., 2015). As a general rule, it is known that glycosylated terpenes are not odorant and form the hidden aroma of grapes. Therefore, their extraction into must during thermomaceration followed by their hydrolysis and conversion into odor-active aglycones during storage can play an important role in the development of enriched juice flavor. However, the increase and de novo synthesis of free and bound aroma compounds after thermomaceration 
Table 1. Aroma compounds in grape musts and enriched juice identified by GC-MS and confirmed by linear retention index (LRI) ${ }^{1}$ values.

\begin{tabular}{|c|c|c|c|c|c|c|c|}
\hline $\mathrm{Nr}$ & Compound & $\operatorname{Prv}^{2}$ & $\mathrm{MSQ}^{3}$ & LRI $^{\mathrm{a}}$ & LRI $^{\mathrm{b}}$ & $\mathrm{CAS}^{4}$ & Odor descriptor $^{5}$ \\
\hline & Terpenoids & & & & & & \\
\hline 1 & D-Limonene & $\mathrm{S}$ & 99 & 1027 & 1027 & $5989-27-5$ & Citrus, sweet, herbal ${ }^{\mathrm{III}}$ \\
\hline \multirow[t]{2}{*}{2} & trans-Geranylacetone & & 95 & 1451 & 1454 & $3796-70-1$ & Green, fruity, rose, waxy, woody, pear, guava ${ }^{\mathrm{V}, \mathrm{VI}}$ \\
\hline & Norisoprenoids & & & & & & \\
\hline 3 & Vitispirane & & 98 & 1279 & 1271 & $65416-59-3$ & Floral $^{\mathrm{III}}$ \\
\hline 4 & Naphthalene, 1,2-dihydro-1,1,6-trimethyl- (TDN) & & 97 & 1351 & 1355 & $30364-38-6$ & Licorice $^{\mathrm{III}}$ \\
\hline \multirow[t]{2}{*}{5} & $\beta$-Damascenone & & 97 & 1384 & 1388 & $23726-93-4$ & Honey, sweet ${ }^{\mathrm{III}}$ \\
\hline & Lactones & & & & & & \\
\hline 6 & 2(5H)-Furanone & $\mathrm{S}$ & 80 & 913 & 916 & $497-23-4$ & Buttery ${ }^{\mathrm{IV}}$ \\
\hline \multirow[t]{2}{*}{7} & $\gamma$-Dodecalactone & & 80 & 1677 & 1681 & $2305-05-7$ & Fatty, fruity, peach, sweet ${ }^{\mathrm{VI}}$ \\
\hline & Aldehydes and ketones & & & & & & \\
\hline 8 & Furfural & $\mathrm{F}$ & 93 & 831 & 835 & 98-01-1 & Sweet, woody, bready, caramel ${ }^{\mathrm{III}}$ \\
\hline 9 & Octanal & $\mathrm{S}$ & 85 & 1002 & 1001 & $124-13-0$ & Waxy, citrus, orange $\mathrm{IV}^{\mathrm{IV}}$ \\
\hline 10 & 2-Nonanone & & 86 & 1090 & 1091 & $821-55-6$ & Fruity, sweet, waxy, soapy, cheese, herbal, coconut ${ }^{\mathrm{V}, \mathrm{VI}}$ \\
\hline 11 & Nonanal & $\mathrm{S}$ & 91 & 1103 & 1102 & $124-19-6$ & Waxy, citrus, cucumber III, IV \\
\hline 12 & Decanal & $\mathrm{S}$ & 91 & 1204 & 1206 & $112-31-2$ & Sweet, citrus, orange, waxy ${ }^{\mathrm{IV}}$ \\
\hline \multirow[t]{2}{*}{13} & 5-Hydroxymethyl-2-furfural & $\mathrm{S}$ & $75-91$ & 1225 & 1230 & $67-47-0$ & Fatty, buttery, musty, waxy, caramel $\mathrm{l}^{\mathrm{VI}}$ \\
\hline & Alcohols & & & & & & \\
\hline 14 & 2-Furanmethanol & $\mathrm{F}$ & 97 & 851 & 853 & $98-00-0$ & Chemical, musty, sweet, caramel, bread, coffee ${ }^{\mathrm{III}}$ \\
\hline 15 & 1-Hexanol & $\mathrm{F}$ & 83 & 865 & 867 & $111-27-3$ & Green, fruity, apple-skin, oily ${ }^{I I I}$ \\
\hline 16 & 2-Ethyl hexanol & & 87 & 1027 & 1027 & $104-76-7$ & Citrus, fresh, floral, oily, sweet ${ }^{\mathrm{V}, \mathrm{VI}}$ \\
\hline 17 & Phenethyl alcohol & $\mathrm{F}$ & 91 & 1111 & 1114 & $60-12-8$ & Floral, sweet, fresh, bready ${ }^{\mathrm{II}}$ \\
\hline 18 & Decanol & $\mathrm{F}$ & 90 & 1271 & 1272 & $112-30-1$ & Sweet, fatty ${ }^{\mathrm{V}}$ \\
\hline \multirow[t]{2}{*}{19} & Dodecanol & & 90 & 1472 & 1475 & $112-53-8$ & Flowery in low concentration $\mathrm{V}$ \\
\hline & Benzenoids & & & & & & \\
\hline 20 & Benzaldehyde & $\mathrm{S}$ & 93 & 958 & 959 & $100-52-7$ & Bitter almond, walnut, smoked ${ }^{\mathrm{III}}$ \\
\hline 21 & Phenol & M & 87 & 979 & 981 & $108-95-2$ & Sweet, tar-like ${ }^{\mathrm{III}, \mathrm{V}}$ \\
\hline 22 & Guaiacol & $\mathrm{S}$ & $76-92$ & 1087 & 1086 & $90-05-1$ & Smoke, sweet, medicine ${ }^{\mathrm{V}}$ \\
\hline 23 & 2,4-Dimethylbenzaldehyde & & 95 & 1183 & 1181 & $15764-16-6$ & Naphthyl, cherry, almond, spice, vanilla ${ }^{\mathrm{VI}}$ \\
\hline 24 & 2-Methoxy-4-vinylphenol & $\mathrm{S}$ & 94 & 1311 & 1313 & 7786-61-0 & Spicy, clove, peanut, woody ${ }^{\mathrm{I}, \mathrm{VI}}$ \\
\hline 25 & Benzaldehyde, 4-hydroxy-3-methoxy (vanillin) & $\mathrm{S}$ & 95 & 1396 & 1395 & $121-33-5$ & Vanilla $^{\mathrm{I}}$ \\
\hline 26 & Isoeugenol & $\mathrm{S}$ & 95 & 1448 & 1456 & $5932-68-3$ & Spicy $^{\mathrm{I}}$ \\
\hline 27 & 2,4-Di-tert-butylphenol & & 97 & 1511 & 1513 & $96-76-4$ & Bilberry, phenolic ${ }^{\mathrm{III}, \mathrm{VI}}$ \\
\hline \multirow[t]{2}{*}{28} & Benzophenone & M & 96 & 1625 & 1623 & 119-61-9 & Balsam, rose, metallic, powdery geranium ${ }^{I I I}$ \\
\hline & Alkenes & & & & & & \\
\hline \multirow[t]{2}{*}{29} & 1-Tetradecene & & 95 & 1390 & 1389 & $1120-36-1$ & Mild, waxy ${ }^{\mathrm{IV}}$ \\
\hline & Acids & & & & & & \\
\hline 30 & Octanoic acid & $\mathrm{F}$ & 80 & 1171 & 1176 & $124-07-2$ & Fatty, waxy, rancid, vegetable, cheese $\mathrm{e}^{\mathrm{II}}$ \\
\hline 31 & Nonanoic acid & $\mathrm{S}$ & 91 & 1269 & 1273 & $112-05-0$ & Waxy, cheese \\
\hline 32 & $n$-Decanoic acid & $\mathrm{F}$ & 97 & 1365 & 1368 & $334-48-5$ & Rancid, sour, fatty, citrus ${ }^{\mathrm{III}}$ \\
\hline 33 & Dodecanoic acid & $\mathrm{S}$ & 98 & 1560 & 1566 & $143-07-7$ & Fatty, coconut, bay oil ${ }^{\mathrm{III}}$ \\
\hline 34 & Tetradecanoic acid & $\mathrm{S}$ & 97 & 1757 & 1763 & $544-63-8$ & Waxy, fatty, soapy, coconut ${ }^{\mathrm{III}}$ \\
\hline 35 & 9-Hexadecenoic acid & & 97 & 1934 & 1942 & $2019-29-4$ & Waxy, creamy, fatty, soapy \\
\hline 36 & Hexadecanoic acid & $\mathrm{S}$ & 99 & 1954 & 1959 & $57-10-3$ & Waxy, fatty ${ }^{\mathrm{III}}$ \\
\hline \multirow[t]{2}{*}{37} & 9,12-Octadecadienoic acid & & 98 & 2145 & 2144 & $60-33-3$ & Fatty ${ }^{I I I}$ \\
\hline & Esters & & & & & & \\
\hline 38 & 1-Butanol, 3-methyl-, acetate & $\mathrm{F}$ & 86 & 875 & 884 & $123-92-2$ & Sweet, banana, fruity ${ }^{\mathrm{III}}$ \\
\hline 39 & Hexyl acetate & $\mathrm{S}$ & 86 & 1013 & 1013 & $142-92-7$ & Green, fruity, sweet, fatty, fresh, apple, pear ${ }^{\mathrm{III}}$ \\
\hline 40 & Ethyl heptanoate & $\mathrm{S}$ & $<70$ & 1098 & 1095 & $106-30-9$ & Fruity, pineapple, sweet, banana ${ }^{\mathrm{III}}$ \\
\hline 41 & Ethyl octanoate & $\mathrm{F}$ & $75-98$ & 1196 & 1196 & $106-32-1$ & Waxy, sweet, musty, pineapple, fruity ${ }^{\mathrm{III}}$ \\
\hline 42 & 2-Phenethyl acetate & $\mathrm{S}$ & 86 & 1256 & 1256 & $103-45-7$ & Fruity, rose, sweet, honey ${ }^{\mathrm{IV}}$ \\
\hline 43 & Ethyl decanoate & $\mathrm{F}$ & 99 & 1394 & 1397 & $110-38-3$ & Sweet, waxy, fruity, apple ${ }^{I I I}$ \\
\hline 44 & Ethyl dodecanoate & $\mathrm{F}$ & 97 & 1593 & 1593 & $106-33-2$ & Sweet ${ }^{\mathrm{V}, \mathrm{VI}}$ \\
\hline 45 & 2-Phenethyl hexanoate & $\mathrm{S}$ & 86 & 1641 & 1639 & $6290-37-5$ & Sweet, honey, floral, waxy ${ }^{\mathrm{VI}}$ \\
\hline 46 & Ethyl tetradecanoate & $\mathrm{F}$ & 96 & 1792 & 1793 & $124-06-1$ & Sweet fruit, butter, fatty ${ }^{\mathrm{VI}}$ \\
\hline 47 & 2-Phenethyl octanoate & $\mathrm{S}$ & 90 & 1845 & 1838 & $5457-70-5$ & Sweet, waxy, green cocoa, fruity ${ }^{\mathrm{VI}}$ \\
\hline 48 & Methyl hexadecanoate & & 98 & 1921 & 1926 & $112-39-0$ & Waxy, fatty, orris ${ }^{\mathrm{VI}}$ \\
\hline 49 & Ethyl E-11-hexadecenoate & & 96 & 1967 & 1974 & $1000245-7-9$ & Waxy, leather ${ }^{\mathrm{VI}}$ \\
\hline 50 & Ethyl hexadecanoate & $\mathrm{F}$ & 99 & 1990 & 1994 & $628-97-7$ & Waxy, fruity, creamy, milky, VI \\
\hline
\end{tabular}

${ }^{1}$ LRI: Linear retention index in a HP-5MS capillary column $\left(30 \mathrm{~m} / 0.25 \mathrm{~mm} / 0.25 \mu \mathrm{m}\right.$, He). LRI ${ }^{\mathrm{a}}$ : Calculated values using van den Dool and Kratz equation. LRI : Data collected from the NIST Chemistry WebBook.

${ }^{2}$ Prv: Standard providers. F: Fluka; M: Merck; S: Sigma-Aldrich.

${ }^{3}$ MS Q: Mass spectrum match quality. This number is a measure of the similarity of the mass spectrum of the compound obtained by sample analysis and those compiled in the NIST or Willey mass spectra libraries. Values near to 100 suggest that the mass spectrum is the same and corresponds to only one compound. ${ }^{4}$ CAS: Chemical Abstracts Service number.

${ }^{5}$ Reference I: Baba and Kumazawa, 2014; II: López de Lerma et al., 2012; III: Vararu et al., 2015; IV: Vararu et al., 2016; V: Welke et al., $2014 b$; VI: TGSC, 2017. 
Table 2. Relative area, standard deviations ( $\mathrm{n}=3$ samples and triplicate analysis) and homogeneous groups (HG) for free aroma compounds.

\begin{tabular}{|c|c|c|c|c|c|c|c|}
\hline \multirow[b]{2}{*}{$\mathrm{Nr}$} & \multirow[b]{2}{*}{ Compound } & \multicolumn{2}{|c|}{ 'País' must } & \multicolumn{2}{|c|}{ 'Lachryma Christi' must } & \multicolumn{2}{|c|}{ Enriched grape juice } \\
\hline & & Mean \pm SD & $\mathrm{HG}$ & Mean \pm SD & $\mathrm{HG}$ & Mean \pm SD & $\mathrm{HG}$ \\
\hline 1 & Terpenoids & $0.170 \pm 0.010$ & $\mathrm{~b}$ & $0.340 \pm 0.030$ & $\mathrm{c}$ & $\mathrm{NF}$ & $\mathrm{a}$ \\
\hline 2 & & $0.118 \pm 0.003$ & $\mathrm{a}$ & $0.120 \pm 0.010$ & $\mathrm{a}$ & $0.130 \pm 0.010$ & $\mathrm{a}$ \\
\hline 3 & Norisoprenoids & $0.210 \pm 0.030$ & $\mathrm{~b}$ & NF & $\mathrm{a}$ & $\mathrm{NF}$ & $\mathrm{a}$ \\
\hline 4 & & $0.260 \pm 0.010$ & $\mathrm{~b}$ & NF & $\mathrm{a}$ & NF & $\mathrm{a}$ \\
\hline 5 & & $0.154 \pm 0.005$ & $\mathrm{~b}$ & NF & $\mathrm{a}$ & NF & $\mathrm{a}$ \\
\hline 6 & Lactones & $0.120 \pm 0.010$ & $\mathrm{~b}$ & $0.079 \pm 0.005$ & $\mathrm{a}$ & $0.170 \pm 0.010$ & $\mathrm{c}$ \\
\hline 7 & & NF & $\mathrm{a}$ & $0.114 \pm 0.010$ & $\mathrm{~b}$ & $\mathrm{NF}$ & $\mathrm{a}$ \\
\hline 8 & Aldehydes and ketones & $0.160 \pm 0.010$ & $\mathrm{~b}$ & $0.100 \pm 0.020$ & $\mathrm{a}$ & $0.180 \pm 0.020$ & $\mathrm{~b}$ \\
\hline 9 & & $0.064 \pm 0.003$ & $\mathrm{a}$ & $0.080 \pm 0.010$ & $\mathrm{~b}$ & $0.078 \pm 0.003$ & $\mathrm{~b}$ \\
\hline 10 & & $0.500 \pm 0.030$ & $\mathrm{~b}$ & NF & $\mathrm{a}$ & NF & $\mathrm{a}$ \\
\hline 11 & & $0.090 \pm 0.010$ & $\mathrm{a}$ & $0.570 \pm 0.040$ & $\mathrm{~b}$ & $0.550 \pm 0.010$ & $\mathrm{~b}$ \\
\hline 12 & & $0.090 \pm 0.010$ & $\mathrm{a}$ & $0.330 \pm 0.020$ & $\mathrm{c}$ & $0.240 \pm 0.020$ & $\mathrm{~b}$ \\
\hline 13 & & $0.006 \pm 0.000$ & $\mathrm{~b}$ & NF & $\mathrm{a}$ & $0.130 \pm 0.010$ & $\mathrm{c}$ \\
\hline 14 & Alcohols & $0.230 \pm 0.010$ & $\mathrm{~b}$ & $0.170 \pm 0.010$ & $\mathrm{a}$ & $0.360 \pm 0.010$ & $\mathrm{c}$ \\
\hline 15 & & $0.212 \pm 0.001$ & $\mathrm{c}$ & $0.170 \pm 0.010$ & $\mathrm{~b}$ & $\mathrm{NF}$ & $\mathrm{a}$ \\
\hline 16 & & $0.042 \pm 0.001$ & $\mathrm{~b}$ & $0.040 \pm 0.010$ & $a b$ & $0.034 \pm 0.002$ & $\mathrm{a}$ \\
\hline 17 & & $0.100 \pm 0.010$ & $\mathrm{~b}$ & NF & $\mathrm{a}$ & $\mathrm{NF}$ & $\mathrm{a}$ \\
\hline 18 & & $0.080 \pm 0.010$ & $\mathrm{~b}$ & $\mathrm{NF}$ & $\mathrm{a}$ & $\mathrm{NF}$ & $\mathrm{a}$ \\
\hline 19 & & $0.096 \pm 0.003$ & $\mathrm{a}$ & $0.140 \pm 0.010$ & $\mathrm{~b}$ & $0.146 \pm 0.001$ & $\mathrm{~b}$ \\
\hline 20 & Benzenoids & $0.030 \pm 0.002$ & $\mathrm{a}$ & $0.040 \pm 0.003$ & $\mathrm{~b}$ & $0.044 \pm 0.003$ & $\mathrm{~b}$ \\
\hline 21 & & NF & $\mathrm{a}$ & NF & $\mathrm{a}$ & $0.080 \pm 0.010$ & $\mathrm{~b}$ \\
\hline 22 & & $0.025 \pm 0.002$ & $\mathrm{a}$ & $0.035 \pm 0.003$ & $\mathrm{~b}$ & $0.032 \pm 0.001$ & $\mathrm{~b}$ \\
\hline 23 & & $0.180 \pm 0.020$ & $\mathrm{a}$ & $0.170 \pm 0.010$ & $\mathrm{a}$ & $0.180 \pm 0.010$ & $\mathrm{a}$ \\
\hline 24 & & $0.045 \pm 0.002$ & $\mathrm{~b}$ & NF & $\mathrm{a}$ & $0.080 \pm 0.010$ & $\mathrm{c}$ \\
\hline 25 & & $\mathrm{NF}$ & $\mathrm{a}$ & $\mathrm{NF}$ & $\mathrm{a}$ & $0.016 \pm 0.001$ & $\mathrm{~b}$ \\
\hline 26 & & $\mathrm{NF}$ & $\mathrm{a}$ & NF & $\mathrm{a}$ & $0.040 \pm 0.003$ & $\mathrm{~b}$ \\
\hline 27 & & $0.100 \pm 0.002$ & $\mathrm{~b}$ & $4.52 \pm 0.19$ & $\mathrm{c}$ & $0.088 \pm 0.002$ & $\mathrm{a}$ \\
\hline 28 & & $0.270 \pm 0.010$ & $\mathrm{~b}$ & $0.210 \pm 0.010$ & $\mathrm{a}$ & $0.220 \pm 0.010$ & $\mathrm{a}$ \\
\hline 29 & Alkenes & $0.100 \pm 0.010$ & $\mathrm{~b}$ & $0.100 \pm 0.010$ & $\mathrm{~b}$ & $0.076 \pm 0.005$ & $\mathrm{a}$ \\
\hline 30 & Acids & $0.260 \pm 0.020$ & $\mathrm{~b}$ & $0.170 \pm 0.020$ & $\mathrm{a}$ & $0.180 \pm 0.020$ & $\mathrm{a}$ \\
\hline 31 & & $0.120 \pm 0.010$ & $\mathrm{a}$ & $0.170 \pm 0.010$ & $\mathrm{~b}$ & $0.160 \pm 0.010$ & $\mathrm{~b}$ \\
\hline 32 & & $0.130 \pm 0.010$ & $\mathrm{~b}$ & $0.090 \pm 0.020$ & $\mathrm{a}$ & $0.080 \pm 0.010$ & $\mathrm{a}$ \\
\hline 33 & & $0.189 \pm 0.005$ & $a b$ & $0.230 \pm 0.020$ & $\mathrm{~b}$ & $0.170 \pm 0.030$ & $\mathrm{a}$ \\
\hline 34 & & $0.085 \pm 0.001$ & $\mathrm{a}$ & $0.140 \pm 0.010$ & $\mathrm{~b}$ & $0.095 \pm 0.005$ & $\mathrm{a}$ \\
\hline 35 & & NF & $\mathrm{a}$ & $0.280 \pm 0.040$ & $\mathrm{~b}$ & $\mathrm{NF}$ & $\mathrm{a}$ \\
\hline 36 & & $0.370 \pm 0.040$ & $\mathrm{a}$ & $1.99 \pm 0.23$ & $\mathrm{c}$ & $1.56 \pm 0.10$ & $\mathrm{~b}$ \\
\hline 37 & & NF & $\mathrm{a}$ & $0.140 \pm 0.070$ & $\mathrm{~b}$ & $0.181 \pm 0.003$ & $\mathrm{~b}$ \\
\hline 38 & Esters & $0.360 \pm 0.020$ & $\mathrm{~b}$ & NF & $\mathrm{a}$ & NF & $\mathrm{a}$ \\
\hline 39 & & $0.150 \pm 0.010$ & $\mathrm{~b}$ & $0.140 \pm 0.020$ & $\mathrm{~b}$ & $\mathrm{NF}$ & $\mathrm{a}$ \\
\hline 40 & & $0.038 \pm 0.001$ & $\mathrm{c}$ & $0.035 \pm 0.003$ & $\mathrm{~b}$ & NF & $\mathrm{a}$ \\
\hline 41 & & $0.055 \pm 0.001$ & $\mathrm{a}$ & $0.080 \pm 0.010$ & $\mathrm{~b}$ & $0.056 \pm 0.002$ & $\mathrm{a}$ \\
\hline 42 & & $0.130 \pm 0.004$ & $\mathrm{~b}$ & $\mathrm{NF}$ & $\mathrm{a}$ & $\mathrm{NF}$ & $\mathrm{a}$ \\
\hline 43 & & $0.270 \pm 0.020$ & $\mathrm{~b}$ & $\mathrm{NF}$ & $\mathrm{a}$ & NF & $\mathrm{a}$ \\
\hline 44 & & $0.300 \pm 0.010$ & $\mathrm{~b}$ & NF & $\mathrm{a}$ & $\mathrm{NF}$ & $\mathrm{a}$ \\
\hline 45 & & $0.296 \pm 0.003$ & $\mathrm{~b}$ & NF & $\mathrm{a}$ & NF & $\mathrm{a}$ \\
\hline 46 & & $0.067 \pm 0.004$ & $\mathrm{~b}$ & $\mathrm{NF}$ & $\mathrm{a}$ & NF & $\mathrm{a}$ \\
\hline 47 & & $0.740 \pm 0.020$ & $\mathrm{~b}$ & NF & $\mathrm{a}$ & NF & $\mathrm{a}$ \\
\hline 48 & & $0.015 \pm 0.001$ & $\mathrm{a}$ & $0.070 \pm 0.010$ & $\mathrm{~b}$ & $0.077 \pm 0.001$ & $\mathrm{~b}$ \\
\hline 49 & & $0.640 \pm 0.040$ & $\mathrm{~b}$ & NF & $\mathrm{a}$ & $\mathrm{NF}$ & $\mathrm{a}$ \\
\hline 50 & & $0.160 \pm 0.010$ & $\mathrm{~b}$ & $\mathrm{NF}$ & $\mathrm{a}$ & $\mathrm{NF}$ & $\mathrm{a}$ \\
\hline
\end{tabular}

For compound details see Table 1.

SD: Standard deviation; NF: not found.

Different letters in the same row indicate significant differences among groups at $\mathrm{p}=0.05$, according to Fisher's least significant difference method.

require further chemometric differentiation between must and juice samples, as well as a statistical selection of key components. This may help to distinguish between process and raw material effects in grape juice enrichment.

\section{Differentiation of grape musts and juice}

Taking into account the diversity of factors that affect the level of each volatile compound in must and juice samples, it is often difficult to interpret volatile data and to establish relationships between chemical constituents, sensory properties and process conditions. However, multivariate data analysis may be useful to group between must samples with and without thermomaceration, and to identify chemical groups and specific aroma compounds that contribute to odor perception of grape musts and enriched juice. Using the data of relative peak area of free aroma compounds, 10 compounds numbered as 1, 6, 12, 13, $14,15,24,27,36$ and 40, show three homogeneous groups (HG) (Table 2). These compounds have been selected for statistical differentiation by cluster analysis (CA) among must and juice samples. On the other hand, nine compounds with numbers $1,3,5,13,20,26,36,37$ and 39 , show 
Table 3. Relative area, standard deviations ( $n=3$ samples and triplicate analysis) and homogeneous groups (HG) for aroma compounds after acidic hydrolysis (free and bound forms).

\begin{tabular}{|c|c|c|c|c|c|c|c|}
\hline \multirow[b]{2}{*}{$\mathrm{Nr}$} & \multirow[b]{2}{*}{ Compound } & \multicolumn{2}{|c|}{ 'País' must } & \multicolumn{2}{|c|}{ 'Lachryma Christi' must } & \multicolumn{2}{|c|}{ Enriched grape juice } \\
\hline & & Mean \pm SD & HG & Mean \pm SD & $\mathrm{HG}$ & Mean \pm SD & $\mathrm{HG}$ \\
\hline 1 & Terpenoids & $0.120 \pm 0.010$ & $\mathrm{a}$ & $0.160 \pm 0.010$ & $\mathrm{~b}$ & $0.220 \pm 0.030$ & $\mathrm{c}$ \\
\hline 2 & & $0.120 \pm 0.010$ & $\mathrm{a}$ & $0.130 \pm 0.010$ & $\mathrm{ab}$ & $0.150 \pm 0.010$ & $\mathrm{~b}$ \\
\hline 3 & Norisoprenoids & $1.21 \pm 0.05$ & $\mathrm{c}$ & $0.570 \pm 0.010$ & $\mathrm{a}$ & $0.810 \pm 0.030$ & $\mathrm{~b}$ \\
\hline 4 & & $0.240 \pm 0.010$ & $\mathrm{a}$ & $0.220 \pm 0.020$ & $\mathrm{a}$ & $0.680 \pm 0.060$ & $\mathrm{~b}$ \\
\hline 5 & & $0.160 \pm 0.010$ & $\mathrm{~b}$ & $0.270 \pm 0.010$ & $\mathrm{c}$ & NF & $\mathrm{a}$ \\
\hline 6 & Lactones & NF & $\mathrm{a}$ & $0.110 \pm 0.020$ & $\mathrm{~b}$ & $0.100 \pm 0.010$ & $\mathrm{~b}$ \\
\hline 8 & Aldehydes and ketones & $0.105 \pm 0.003$ & $\mathrm{a}$ & $0.160 \pm 0.040$ & $\mathrm{~b}$ & $0.160 \pm 0.010$ & $\mathrm{~b}$ \\
\hline 9 & & $0.070 \pm 0.010$ & $\mathrm{a}$ & $0.090 \pm 0.010$ & $\mathrm{~b}$ & $0.070 \pm 0.010$ & $\mathrm{a}$ \\
\hline 10 & & $0.270 \pm 0.030$ & $\mathrm{~b}$ & NF & $\mathrm{a}$ & NF & $\mathrm{a}$ \\
\hline 11 & & $0.480 \pm 0.030$ & $\mathrm{a}$ & $0.560 \pm 0.030$ & $\mathrm{ab}$ & $0.640 \pm 0.100$ & $\mathrm{~b}$ \\
\hline 12 & & $0.150 \pm 0.010$ & $\mathrm{a}$ & $0.210 \pm 0.010$ & $\mathrm{~b}$ & $0.240 \pm 0.050$ & $\mathrm{~b}$ \\
\hline 13 & & NF & $\mathrm{a}$ & $0.110 \pm 0.010$ & $\mathrm{~b}$ & $0.130 \pm 0.020$ & $\mathrm{c}$ \\
\hline 14 & Alcohols & $0.131 \pm 0.005$ & $\mathrm{a}$ & $0.220 \pm 0010$ & $\mathrm{~b}$ & $0.230 \pm 0.020$ & $\mathrm{~b}$ \\
\hline 15 & & $0.210 \pm 0.020$ & $\mathrm{~b}$ & $0.220 \pm 0.010$ & $\mathrm{~b}$ & NF & $\mathrm{a}$ \\
\hline 16 & & NF & $\mathrm{a}$ & NF & $\mathrm{a}$ & $0.040 \pm 0.020$ & $\mathrm{~b}$ \\
\hline 17 & & $0.110 \pm 0.010$ & $\mathrm{~b}$ & NF & $\mathrm{a}$ & NF & $\mathrm{a}$ \\
\hline 18 & & $0.072 \pm 0.004$ & $\mathrm{~b}$ & NF & $\mathrm{a}$ & $\mathrm{NF}$ & $\mathrm{a}$ \\
\hline 19 & & $0.078 \pm 0.004$ & $\mathrm{~b}$ & NF & $\mathrm{a}$ & $0.100 \pm 0.020$ & $\mathrm{~b}$ \\
\hline 20 & Benzenoids & $0.060 \pm 0.010$ & $\mathrm{~b}$ & $0.029 \pm 0.001$ & $\mathrm{a}$ & $0.390 \pm 0.004$ & $\mathrm{c}$ \\
\hline 22 & & NF & $\mathrm{a}$ & $0.039 \pm 0.002$ & $\mathrm{~b}$ & NF & $\mathrm{a}$ \\
\hline 23 & & $0.148 \pm 0.004$ & $\mathrm{a}$ & $0.168 \pm 0.008$ & $\mathrm{~b}$ & $0.170 \pm 0.010$ & $\mathrm{~b}$ \\
\hline 24 & & $0.052 \pm 0.000$ & $\mathrm{a}$ & $0.091 \pm 0.006$ & $\mathrm{~b}$ & $0.100 \pm 0.010$ & $\mathrm{~b}$ \\
\hline 25 & & NF & $\mathrm{a}$ & $0.032 \pm 0.004$ & $\mathrm{~b}$ & $0.040 \pm 0.010$ & $\mathrm{~b}$ \\
\hline 26 & & $\mathrm{NF}$ & $\mathrm{a}$ & $0.048 \pm 0.004$ & $\mathrm{~b}$ & $0.100 \pm 0.010$ & $\mathrm{c}$ \\
\hline 27 & & $0.082 \pm 0.004$ & $\mathrm{a}$ & $0.088 \pm 0.004$ & $\mathrm{a}$ & $0.080 \pm 0.010$ & $\mathrm{a}$ \\
\hline 28 & & $0.250 \pm 0.030$ & $\mathrm{a}$ & $0.246 \pm 0.020$ & $\mathrm{a}$ & $0.240 \pm 0.020$ & $\mathrm{a}$ \\
\hline 29 & Alkenes & $0.050 \pm 0.010$ & $\mathrm{~b}$ & $0.050 \pm 0.010$ & $\mathrm{~b}$ & NF & $\mathrm{a}$ \\
\hline 30 & Acids & $0.110 \pm 0.010$ & $\mathrm{a}$ & $0.130 \pm 0.010$ & $\mathrm{a}$ & $0.120 \pm 0.010$ & $\mathrm{a}$ \\
\hline 31 & & $0.090 \pm 0.020$ & $\mathrm{a}$ & $0.090 \pm 0.010$ & $\mathrm{a}$ & $0.120 \pm 0.010$ & $\mathrm{a}$ \\
\hline 32 & & $0.130 \pm 0.020$ & $\mathrm{~b}$ & $0.050 \pm 0.010$ & $\mathrm{a}$ & $0.050 \pm 0.010$ & $\mathrm{a}$ \\
\hline 33 & & $0.210 \pm 0.010$ & $\mathrm{~b}$ & $0.120 \pm 0.020$ & $\mathrm{a}$ & $0.180 \pm 0.020$ & $\mathrm{~b}$ \\
\hline 34 & & $0.100 \pm 0.003$ & $\mathrm{a}$ & $0.110 \pm 0.010$ & $\mathrm{a}$ & $0.110 \pm 0.020$ & $\mathrm{a}$ \\
\hline 35 & & $\mathrm{NF}$ & $\mathrm{a}$ & $0.970 \pm 0.080$ & $\mathrm{~b}$ & $\mathrm{NF}$ & $\mathrm{a}$ \\
\hline 36 & & $0.370 \pm 0.040$ & $\mathrm{a}$ & $0.850 \pm 0.140$ & $\mathrm{~b}$ & $1.18 \pm 0.31$ & $\mathrm{c}$ \\
\hline 37 & & $\mathrm{NF}$ & $\mathrm{a}$ & $0.064 \pm 0.002$ & $\mathrm{~b}$ & $0.090 \pm 0.020$ & $\mathrm{c}$ \\
\hline 38 & Esters & $0.190 \pm 0.020$ & $\mathrm{~b}$ & NF & $\mathrm{a}$ & NF & $\mathrm{a}$ \\
\hline 39 & & $0.200 \pm 0.010$ & $\mathrm{c}$ & $0.119 \pm 0.004$ & $\mathrm{~b}$ & $\mathrm{NF}$ & $\mathrm{a}$ \\
\hline 40 & & $0.042 \pm 0.004$ & $\mathrm{a}$ & $0.070 \pm 0.010$ & $\mathrm{~b}$ & $0.080 \pm 0.010$ & $\mathrm{~b}$ \\
\hline 41 & & $0.160 \pm 0.010$ & $\mathrm{a}$ & $0.155 \pm 0.000$ & $\mathrm{a}$ & $0.170 \pm 0.020$ & $\mathrm{a}$ \\
\hline 42 & & $0.072 \pm 0.004$ & $\mathrm{~b}$ & NF & $\mathrm{a}$ & NF & $\mathrm{a}$ \\
\hline 48 & & $0.060 \pm 0.001$ & $\mathrm{a}$ & $0.070 \pm 0.002$ & $\mathrm{~b}$ & $0.080 \pm 0.010$ & $\mathrm{~b}$ \\
\hline
\end{tabular}

For compound details see Table 1.

SD: Standard deviation; NF: not found.

Different letters in the same row indicate significant differences among groups at $\mathrm{p}=0.05$, according to Fisher's least significant difference method.

significant differences $(\mathrm{p}<0.05)$ among the three samples (PA, LC and EJ) according to the data of relative peak area of free and bound aroma compounds that have been selected for further differentiation among the samples (Table 3). According to Figure 1a, PA and LC musts show more similarity with regard to free aroma compounds compared to enriched juice. On the other hand, chemical composition of PA must for both free and bound aroma compounds is different compared to LC and EJ samples (Figure 1b). Thermomaceration of grape pomace, leaves and canes in must modifies sensory profile by free aroma compounds and to a less extent the global aroma compounds detected after hydrolysis. Apparently, thermomaceration is unable to provoke the hydrolysis of a large amount of bound aroma compounds.

After statistical treatment by MVA of analytical data of volatile compounds previously classified in chemical families, the resulting Sunray Plots (Statgraphics Plus, 2.0,
STSC Inc., Rockville, Maryland, USA; Figure 2) yield a visual overview of free and total aroma development from grape musts to enriched juice. In here, each ray in the polygon corresponds to a chemical family. The distance from the center to each vertex corresponds to the value of each group. The end of the ray is the mean value plus three standard deviations and the center the mean minus three standard deviations. Concerning free aroma compounds, both PA and LC musts exhibit irregular polygons, while a relatively regular polygon structure was found for the EJ sample (Figure 2). These fingerprints show high ester values for PA. These compounds are related to odor descriptors that result into a pleasant fruit-like odor, while benzenoids prevail in LC are related to a phenolic, balsamic odor. Fingerprints differ also for aroma compounds after acidic hydrolysis. In this case, a more regular polygon structure can be observed for LC must (Figure 2). Fatty acids with an unpleasant rancid odor are predominant in LC and enriched 
Figure 1. Dendrograms resulting from applying cluster analysis to the selected aroma compounds as: Free aroma compounds (a) and total content (b) of aroma compounds after acidic hydrolysis.

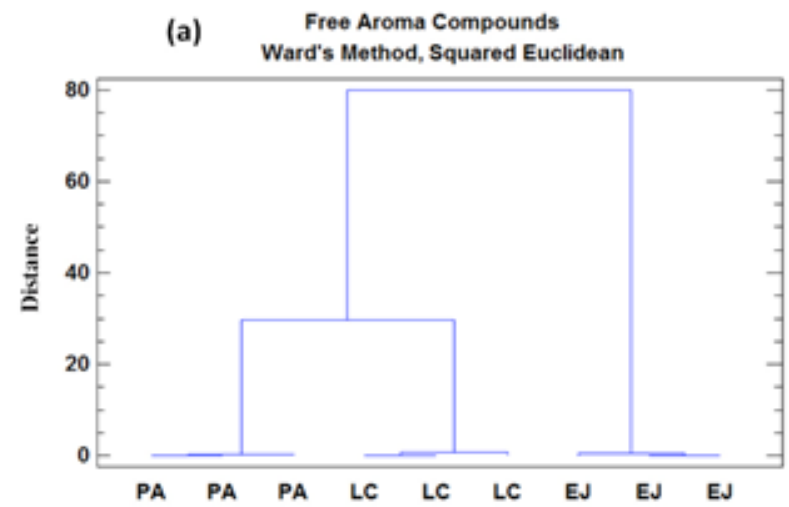

(b)

Aroma compounds after acidic hydrolysis

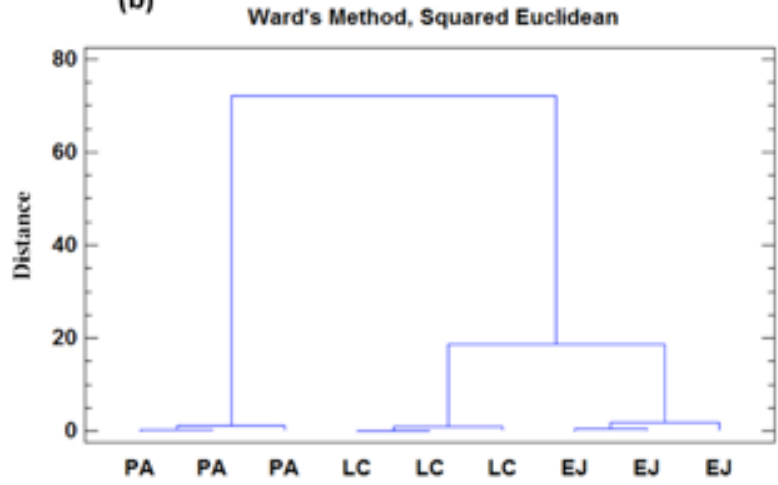

PA: ‘País’ must; LC: ‘Lachryma Christi’ must; EJ: Enriched grape juice.

juice, while norisoprenoids with a floral odor prevail in PA must. These results are in accordance with Vararu et al. (2015) who found that fingerprints depend upon cultivars and grape-growing area.
In order to clarify differences among grape musts and enriched juice, and identify constituents able to distinguish thermomaceration from control treatments, a principal component analysis was carried out using as grouping variables those aroma compounds with different homogeneous groups. According to Figure 3a, two principal components (PC) accounted for $99.10 \%$ of the total variance of free aroma compounds. For the first PC, four free aroma compounds (D-limonene (1), 1-hexanol (15), 2,4-di-tert-butylphenol (27) and ethyl heptanoate (40)) have been selected due to their positive weight coefficients higher than 0.3 , while the compounds $2(5 H)$-furanone (6), 5-hydroxymethyl-2-furfural (13), 2-furanmethanol (14) and 2-methoxy-4-vinylphenol (24) selected because of their negative weight coefficients less than -0.3 . For the second PC, decanal (12), 1-hexanol (15), 2,4-di-tertbutylphenol (27) and hexadecanoic acid (36) show the highest contribution in must characterization. Then, PC1 and PC2 scores group enriched grape juice and musts of both grape cultivars. 'Lachryma Christi' must differs from PA must and EJ sample by a higher score on PC1, while PA must is differentiated by its negative PC2 score (Figure $3 a)$. Free compounds that considerably contribute to the aroma profile of LC must are 2,4-di-tert-butylphenol (27) with bilberry and D-limonene (1) with citrus, sweet and herbal odor descriptors. Free 5-hydroxymethyl-2-furfural (13) yields a significant contribution to the aroma profile of enriched grape juice, which indicates the importance of heat treatment for juice processing. The free aroma profile of PA must is more complex, but should include those compounds with a negative weight coefficient for PC2 (1-hexanol (15) and ethyl heptanoate (40)). On the other hand, PCA done with nine compounds selected after acidic hydrolysis of musts and juice provided two principal components: PC1 with an eigenvalue of 6.86 and PC2 with 1.97 , explaining $76.25 \%$ and $21.88 \%$ of the total variance,

Figure 2. Grape musts fingerprint obtained by multivariate data analysis of aroma compounds as free forms (I series) and after acidic hydrolysis (II series), grouped by chemical families.

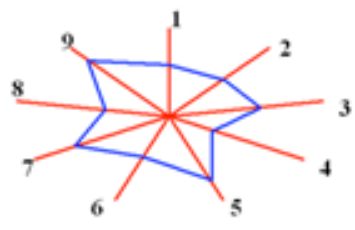

$P A(\mathrm{I})$

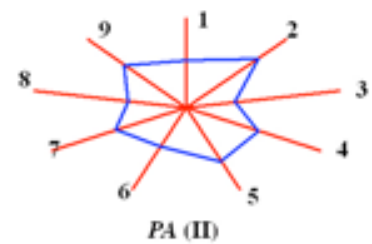

PA (II)
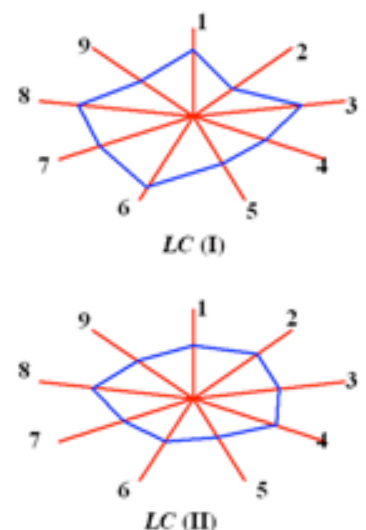
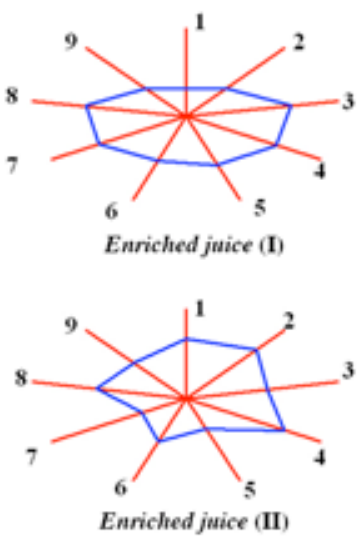

PA: 'País' must; LC: 'Lachryma Christi' must.

Each number corresponds to a chemical family: 1. Terpenoids; 2. Norisoprenoids; 3. Lactones; 4. Aldehydes and ketones; 5. Alcohols; 6. Benzenoid compounds; 7. Alkenes; 8. Acids; 9. Esters. 
Figure 3. Principal component analyses of selected aroma compounds as free forms (a) and after acidic hydrolysis (b). For compound numbers see Table 1.

(a) Free Aroma Compounds ( $99.10 \%$ of total variance)

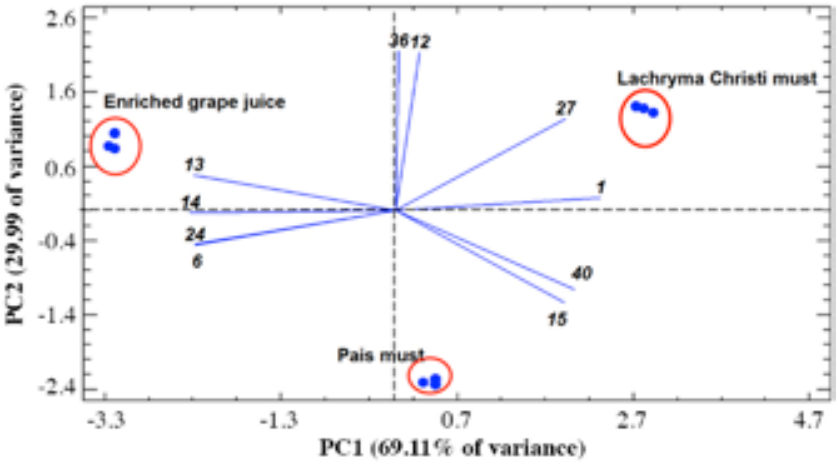

(b) Aroma compounds after acidic hydrolysis ( $98.13 \%$ of total variance)

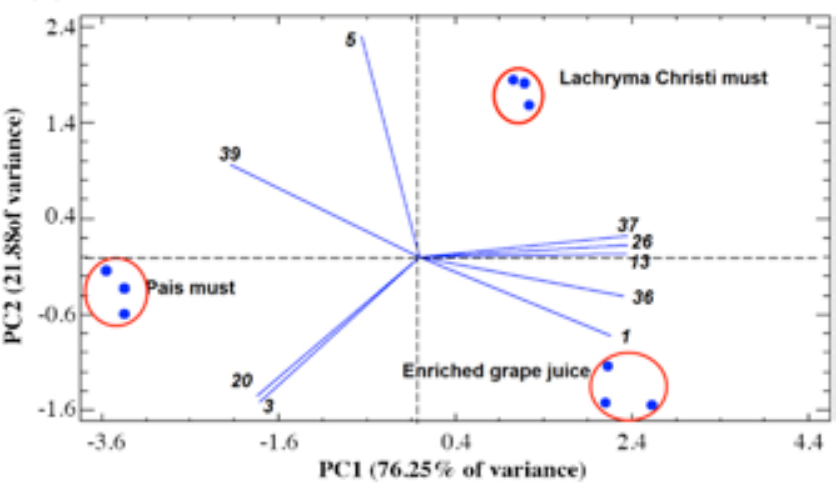

respectively. The most important free and compounds after acidic hydrolysis that contribute to $\mathrm{PC} 1$ with a positive weight coefficient are D-limonene (1), 5-hydroxymethyl2-furfural (13), isoeugenol (26), hexadecanoic acid (36) and 9,12-octadecadienoic acid (37). Just hexyl acetate (39) contributes to PC1 with a negative weight coefficient. The most important free and bound aroma compounds for PC2 are vitispirane (3), $\beta$-damascenone (5) and benzaldehyde (20) with weight coefficients of $-0.446,0.679$ and -0.425 , respectively. According to Figure $3 \mathrm{~b}, \mathrm{LC}$ and $\mathrm{EJ}$ are located to the right, showing a positive score for PC1, while PA is located at the left side. LC and EJ have opposite scores for PC2, while PA shows values near to zero for PC2. After hydrolysis, D-limonene (1) is the most characteristic aroma compound for enriched grape juice (Figure $3 b$ ). None of the selected aroma compounds after hydrolysis could be individualized and associated directly to LC or PA must. The results obtained by means of PCA after acidic hydrolysis show the complexity of aroma potential of PA and LC musts.

According to previous results chemometrics can be successfully applied to characterize grape juice samples after thermomaceration being D-limonene, 5-hydroxymethyl-2-furfural and hexadecanoic acid the compounds that contributed most to the free aroma fraction and the fraction obtained after hydrolysis. In addition, 5-hydroxymethyl-2-furfural, a key component of the free aroma fraction of enriched juice, seems to be related to juice processing conditions. Heating of monosaccharides under acidic conditions e.g. pasteurization of fruit juices gives rise to a large number of furan compounds, amongst them 5-hydroxymethyl-2-furfural (Belitz et al., 2009), which agrees with the results of this study (Table 2). Another key component is hexadecanoic acid that may become available after grape crushing due to the contact between must and skins, particularly, in case of 'Lachryma Christi' grapes. Esters of hexadecanoic acids are the major part of cuticular waxes of red grape skins (Mendes et al., 2013), which may be converted into the free acid form during juice processing and thermomaceration.

\section{CONCLUSIONS}

Since viticulturists are looking for new productive alternatives for ancient undervalued wine grape cultivars, this study provides new strategies for their valorization by revealing the chemical composition and aroma potential of grape musts and winery and grapevine by-products. In particular, chemometrics applied to aroma data using SBSE-TD-GC-MS with and without hydrolysis are very useful for the characterization of pure grape juices of País and Lachryma Christi cultivars and the resulting juice from thermomaceration. In addition, grape pomace, grapevine leaves and canes are valuable sources to yield aroma enrichment of grape juice or the generation of new aroma compounds. Juice processing conditions affect the rather complex aroma profile of enriched grape juice.

\section{ACKNOWLEDGEMENTS}

The authors wish to thank Viña Zamora for providing grape samples, and the Chilean Ministry of Education (FONDEF VIU 120010) and the European Union (Erasmus + KA1) for financial support.

\section{REFERENCES}

Aguilar, T., Loyola, C., de Bruijn, J., Bustamante, L., Vergara, C., von Baer, D., et al. 2016. Effect of thermomaceration and enzymatic maceration on phenolic compounds of grape must enriched by grape pomace, vine leaves and canes. European Food Research and Technology 242:1149-1158. doi:10.1007/ s00217-015-2619-3.

Baba, R., and Kumazawa, K. 2014. Characterization of the potent odorants contributing to the characteristic aroma of Chinese green tea infusions by aroma extract dilution analysis. Journal of Agricultural and Food Chemistry 62:8308-8313. doi:10.1021/jf502308a.

Baumes, R. 2009. Wine aroma precursors. p. 251-274. In MorenoArribas, M.V., and Polo, M.C. (eds.) Wine chemistry and biochemistry. Springer, New York, USA.

Belitz, H.D., Grosch, W., and Schieberle, P. 2009. Food chemistry. $4^{\text {th }}$ ed. Springer, Berlin, Germany.

Ferrandino, A., Carlomagno, A., Baldassarre, S., and Schubert, A. 2012. Varietal and pre-fermentative volatiles during ripening of Vitis vinifera cv. Nebbiolo berries from three growing areas. Food Chemistry 135:2340-2349. doi:10.1016/ j.foodchem.2012.06.061. 
Galano, E., Imbelloni, M., Chambery, A., Malorni, A., and Amoresano, A. 2015. Molecular fingerprint of the alcoholic Grappa beverage by mass spectrometry techniques. Food Research International 72:106-114. doi:10.1016/ j.foodres.2015.03.033.

Ghaste, M., Narduzzi, L., Carlin, S., Vrhovsek, U., Shulaev, V., and Mattivi, F. 2015. Chemical composition of volatile aroma metabolites and their glycosylated precursors that can unique differentiate individual grape. Food Chemistry 188:309-319. doi:10.1016/j.foodchem.2015.04.056.

González-Barreiro, C., Rial-Otero, R., Cancho-Grande, B., and Simal-Gándara, J. 2015. Wine aroma compounds in grapes: A critical review. Critical Reviews in Food Science and Nutrition 55:202-218. doi:10.1080/10408398.2011.650336.

Iyer, M.M., Sacks, G.L., and Padilla-Zakour, O.I. 2010. Impact of harvesting and processing conditions on green leaf volatile development and phenolics in Concord grape juice. Journal of Food Science 75:C297-C304. doi:10.1111/j.17503841.2010.01559.x.

López de Lerma, N., García-Martínez, T., Moreno, J., Mauricio, J.C., and Peinado, R.A. 2012. Volatile composition of partially fermented wines elaborated from sun dried Pedro Ximénez grapes. Food Chemistry 135:2445-2452. doi:10.1016/ j.foodchem.2012.07.058.

Martínez-Gil, A.M., Garde-Cerdán, T., Martínez, L., Alonso, G.L., and Salinas, M.R. 2011. Effect of oak extract application to Verdejo grapevines on grape and wine aroma. Journal of Agricultural and Food Chemistry 59:3253-3263. doi:10.1021/ jf104178c.

Mendes, J.A.S., Prozil, S.O., Evtuguin, D.V., and Cruz Lopes, L.P. 2013. Towards comprehensive utilization of winemaking residues: Characterization of grape skins from red grape pomaces of variety Touriga Nacional. Industrial Crops and Products 43:25-32. doi:10.1016/j.indcrop.2012.06.047.

Milla Tapia, A., Cabezas, J.A., Cabello, F., Lacombe, T., MartínezZapater, J.M., Hinrichsen, P., et al. 2007. Determining the Spanish origin of representative ancient American grapevine varieties. American Journal of Enology and Viticulture 58:242251.

NIST. 2016. Web book of chemistry. National Institute of Standards and Technology (NIST). Available at http://webbook. nist.gov/chemistry/ (accessed September 2016).

Noguerol-Pato, R., González-Barreiro, C., Cancho-Grande, B., Santiago, J.L., Martínez, M.C., and Simal-Gándara, J. 2012. Aroma potential of Brancellao grapes from different cluster positions. Food Chemistry 132:112-124. doi:10.1016/ j.foodchem.2011.10.042

Pedroza, M.A., Carmona, M., Alonso, G.L., and Salinas, M.R. 2013. Pre-bottling use of dehydrated waste grape skins to improve color, phenolic and aroma composition of red wines. Food Chemistry 136:224-236. doi:10.1016/ j.foodchem.2012.07.110.

Pedroza, M.A., Zalacain, A., Lara, J.F., and Salinas, M.R. 2010. Global grape aroma potential and its individual analysis by SBSE-GC-MS. Food Research International 43:1003-1008. doi:10.1016/j.foodres .2010.01.008.

Seisonen, S., Vene, K., and Koppel, K. 2016. The current practice in the application of chemometrics for correlation of sensory and gas chromatography data. Food Chemistry 210:530-540. doi:10.1016/j.foodchem.2016.04.134.
Slegers, A., Angers, P., Ouellet, E., Truchon, T., and Pedneault, K. 2015. Volatile compounds from grape skin, juice and wine from five interspecific hybrid grape cultivars grown in Québec (Canada) for wine production. Molecules 20:10980-11016. doi:10.390/molecules200610980.

Statistica. 2016. Statistica - the portal for statistics. Statistica, New York, USA. Available at https://www.statistica.com/ outlook/20030500/102/grape-juice/europe\# (accessed November 2016).

Teixeira, A., Baenas, N., Dominguez-Perles, R., Barros, A., Rosa, E., Moreno, D.A., et al. 2014. Natural bioactive compounds from winery by-products as health promoters: a review. International Journal of Molecular Sciences 15:15638-15678. doi:10.3390/ijms 150915638 .

TGSC. 2017. The Good Scents Company Information System. Available at http://www.thegoodscentscompany.com (accessed May 2017).

Vararu, F., Moreno-Garcia, J., Cotea, V.V., and Moreno, J. 2015. Grape musts differentiation based on selected aroma compounds using SBSE-GC-MS and statistical analysis. Vitis 54:97-105. Available at http://pub.jki.bund.de/index.php/VITIS/article/ view/5288 (accessed May 2017).

Vararu, F., Moreno-Garcia, J., Zamfir, C.I., Cotea, V.V., and Moreno, J. 2016. Selection of aroma compounds for the differentiation of wines obtained by fermenting musts with starts cultures of commercial yeast strains. Food Chemistry 197:373381. doi:10.1016/j.foodchem.2015.10.111.

Wang, X.J., Tao, Y.S., Wu, Y., An, R.Y., and Yue, Z.Y. 2017. Aroma compounds and characteristics of noble-rot wines of Chardonnay grapes artificially botrytized in the vineyard. Food Chemistry 226:41-50. doi:10.1016/j.foodchem.2017.01.007.

Ward, S.C., Petrie, P.R., Johnson, T.E., Boss, P.K., and Bastian, S.E.P. 2015. Unripe berries and petioles in Vitis vinifera $\mathrm{cv}$. Cabernet Sauvignon fermentations affect sensory and chemical profiles. American Journal of Enology and Viticulture 66:435443. doi:10.5344/ajev.2015.15016.

Weldegergis, B.T., de Villiers, A., and Crouch, A.M. 2011. Chemometric investigation of the volatile content of young South African wines. Food Chemistry 128:1100-1109. doi:10.1016/j.foodchem.2010.09.100.

Welke, J.E., Zanus, M., Lazzarotto, M., and Alcaraz-Zini, C. 2014b. Quantitative analysis of headspace volatile compounds using comprehensive two-dimensional gas chromatography and their contribution to the aroma of Chardonnay wine. Food Research International 59:85-99. doi:10.1016/ j.foodres.2014.02.002.

Welke, J.E., Zanus, M., Lazzarotto, M., Hepp-Pulgati, F., and Alcaraz-Zini, C. 2014a. Main differences between volatiles of sparkling and base wines accessed through comprehensive two dimensional gas chromatography with time-of-flight spectrometric detection and chemometric tools. Food Chemistry 164:427-437. doi:10.1016/j.foodchem.2014.05.025.

Yuan, F., and Qian, M.C. 2016. Aroma potential in early- and latematurity Pinot noir grapes evaluated by aroma extract dilution analysis. Journal of Agricultural and Food Chemistry 64:443450. doi:10.1021/acs.jafc.5b04774. 